\title{
Éric Sabourin - Organisations et sociétés paysannes. Une lecture par la réciprocité - éditions Quae, paris, 2012, 262 pages
}

\section{Rémy Herrera}

\section{(2) OpenEdition}

\section{Journals}

Electronic version

URL: http://journals.openedition.org/economierurale/4411

DOI: 10.4000/economierurale.4411

ISSN: 2105-2581

Publisher

Société Française d'Économie Rurale (SFER)

\section{Printed version}

Date of publication: 15 July 2014

Number of pages: 121-123

ISSN: 0013-0559

\section{Electronic reference}

Rémy Herrera, «Éric Sabourin - Organisations et sociétés paysannes. Une lecture par la réciprocité éditions Quae, paris, 2012, 262 pages », Économie rurale [En ligne], 342 | juillet-août 2014, mis en ligne le 15 juillet 2014, consulté le 24 septembre 2020. URL : http://journals.openedition.org/ economierurale/4411 ; DOI : https://doi.org/10.4000/economierurale.4411 
ÉRIC SABOURIN

\section{Organisations et sociétés paysannes \\ Une lecture par la réciprocité}

Versailles, Quae, 2012, XVI-262 p.
A vec Organisations et sociétés paysannes: une lecture par la réciprocité, Éric Sabourin, socio-anthropologue au département Environnement et Sociétés du Cirad et professeur visitant à l'Université de Brasilia depuis septembre 2012, nous offre un livre de synthèse fort intéressant. L'ambition de l'ouvrage, qui s'ouvre par une érudite préface du professeur Jan Douwe van der Ploeg de I'Université de Wageningen, est d'utiliser le cadre de la théorie de la réciprocité pour une analyse des évolutions des sociétés paysannes - en les situant au croisement du fonctionnement des marchés et des interventions de l'État.

S'appuyant continûment et plutôt solidement sur des éléments théoriques, l'auteur s'efforce d'appliquer cette problématique axée sur la réciprocité, qu'il étudie depuis la fin des années 1970, à plusieurs études de cas portant sur des régions du monde très diverses (francophones, hispanophones et lusophones), couvrant presque tous les continents. Un point fort du livre est son interdisciplinarité, faisant dialoguer avec un certain succès l'anthropologie, la sociologie et l'économie.

La première partie présente l'intérêt des approches menées selon la théorie de la réciprocité en sciences sociales, en se référant aux apports d'auteurs fondamentaux (notamment simmel, Mauss, Lévi-Strauss ou Polanyi) ou bien plus récents (comme Temple et Chabal, entre autres). L'auteur analyse ainsi la construction de cette théorie, ses logiques, ses formes, ses structures, tout en présentant dans le même temps les critiques qui lui sont adressées (quant à la primauté du don en particulier), les limites qui la caractérisent (par exemple, celles relatives à la logique du tiers inclus ou à la production de valeurs), ainsi que certaines des pistes qui pourraient en prolonger - d'après l'auteur, fructueusement - les perspectives (y compris dans des systèmes qualifiés de " mixtes", où coexistent relations de réciprocité et relations d'échange).

En alternant réflexion théorique et études empiriques, appliquées principalement à l'Afrique (avec plusieurs cas proposés sur la Guinée-Bissau : les riziculteurs balantes de Tombali, les Mandjaks de Cachungo...), la deuxième partie du livre revient sur l'examen du principe de réciprocité dans les organisations, les communautés et les sociétés paysannes. Les repères théoriques sont alors placés en interaction avec des analyses conduites en termes de gouvernance des communs (Ostrom), d'économie rurale (Scott) ou d'économie de l'affection (Hyden). Éric Sabourin, dans son élément - la socio-anthropologie -, démontre sur ces bases la persistance de pratiques d'entraide rurale, de gestion des ressources communes et de formes d'organisation fondées sur les relations de réciprocité.

Ces dernières sont ensuite étudiées, dans une troisième partie, intitulée "Les organisations paysannes face au marché et à l'État ", dans les transactions de redistribution et de commercialisation. Les distinctions établies entre l'échange et la réciprocité sont ici identifiées à partir de l'étude de l'origine et du fonctionnement de certains marchés africains, mais aussi des marchés kanaks de NouvelleCalédonie. Les investigations de l'auteur en économie solidaire l'amènent par là à d'intéressants développements sur les limites du " commerce équitable ".

Puis, toujours en s'appuyant sur des cas concrets (la pêche artisanale aux îles Bijagos de Guinée-Bissau, par exemple), Éric Sabourin en vient à des essais d'évaluation des politiques publiques dite 
" d'interface " (c'est-à-dire opérant entre logique de l'échange marchand et logique de réciprocité), ainsi que de celle de l'influence des organisations paysannes sur l'évolution des politiques publiques territoriales et de développement rural. Sont aussi étudiées les législations foncières et les lois relatives à certaines communautés autochtones, du cas des communautés indigènes du Pérou à celui des avancées produites en la matière par les nouvelles constitutions en Équateur et en Bolivie.

L'ouvrage se termine par quelques considérations relatives aux divers risques dits "d'aliénation " de la logique de réciprocité, notamment sous les formes d'asymétrie et de dépendance, de clientélisme et de corruption, ou de paternalisme et de "patrimonialisme " - ou même encore de diverses variantes du " populisme ". C'est alors surtout la sociologie politique qui est mobilisée, parmi d'autres champs disciplinaires ; et le continent latino-américain, à commencer par plusieurs régions du Brésil, qui sert ici d'application à ces réflexions.

L'apport de ce livre est d'un intérêt réel, tout spécialement par la synthèse qu'il fournit et par la diversité des études de cas qu'il propose, pour une analyse des liaisons complexes entre la théorie de la réciprocité et la question paysanne. La seule petite frustration que nous laisse, personnellement, la lecture de cet ouvrage d'Éric Sabourin est la réticence de ce dernier, pour ce qu'il nous a semblé, à intégrer plus amplement dans ses référents analytiques comme dans ses argumentations appliquées les contributions théoriques ou empiriques de certains courants connexes - marxistes, entre autres, et pour être clair -, dont il paraît pourtant proche, mais auxquels il ne renvoie que fort rarement.

Pour ne donner ici qu'un simple exemple, l'interprétation - à notre avis lapidaire - qui est donnée par l'auteur (hélas, essentiellement à partir de textes de seconde main) des positions, des méthodes et des propositions du
Mouvement des travailleurs sans terre (MST) du Brésil aurait très probablement gagné en pertinence si elle avait reposé sur une analyse précise et approfondie de la littérature, abondante et de qualité, produite par cette organisation de masse, notamment certains de ses intellectuels (João Pedro Stédile, par exemple), qui ne sont pas que des activistes, mais également des théoriciens dont les écrits s'avèrent extrêmement utiles pour les chercheurs scientifiques et les décideurs politiques.

Car on comprend difficilement la radicalité d'un tel mouvement - qui est d'ailleurs loin d'être le seul à souligner la nécessité d'une réforme agraire et à revendiquer des changements structurels pour les sociétés du Sud - si l'on ne replace pas ses luttes dans le contexte des formes d'activité et de prise de contrôle, singulièrement violentes, des firmes transnationales et du capital financier sur ces continents en développement. Ce qu'il s'agirait selon nous de bien saisir, ce sont les logiques et mécanismes par lesquels ce capital opère aujourd'hui dans le secteur agricole, durant sa phase de domination du capital financier, ainsi que les caractéristiques spécifiques générées par la récente crise systémique et de leurs conséquences pour l'organisation de la production agricole comme pour la vie elle-même des paysans dans les pays étudiés. Sans économicisme, l'interdisciplinarité aurait peut-être mérité d'être poussée jusque-là...

De façon plus générale, la réduction de cette distanciation vis-à-vis des courants de pensée mentionnés aurait sans doute aussi permis de différencier plus nettement échanges marchands et échanges marchands capitalistes ; comme également d'investir plus profondément les questions de la gestion démocratique des ressources naturelles et de l'écologie politique; ou encore de mesurer à leur plus juste valeur l'ampleur de luttes populaires et des avancées révolutionnaires en cours - en Amérique latine, dans le 
cas qui occupe Éric Sabourin -, ainsi que leur importance pour l'accès à la terre et à l'amélioration des conditions de vie et de travail des familles paysannes, que nous sentons si chères au cœur de l'auteur.

Ces quelques considérations finales, toutes personnelles et bienveillantes, n'enlèvent rien aux qualités multiples et tout à fait remarquables de cet ouvrage - dont nous saluons la richesse et la technicité, et que nous recommandons vivement aux lecteurs d'Économie rurale.

Rémy HERRERA

Université de Paris I, CNRS, UMR 8174 Centre d'Économie de la Sorbonne 